\title{
Ultrasound measurement of renal size in newborn infants
}

\author{
J E S Scott, E W Hunter, R E J Lee, J N S Matthews
}

\begin{abstract}
Renal measurements were made by ultrasonography in $\mathbf{5 6 0}$ healthy newborn infants. Three dependent variables (kidney length, kidney depth, and kidney area) were measured, together with three independent variables (birth weight, head circumference, and gestational age).
\end{abstract}

The SD of the measurement inconsistency was $0.13 \mathrm{~cm}$ for length, $0.078 \mathrm{~cm}$ for depth, and $0.32 \mathrm{~cm}^{2}$ for area; the percentages of total variance caused by measurement inconsistency were $7.9,9.5$, and $7 \cdot 5$, respectively. There was a significant difference between right and left kidneys in length and depth for each independent variable. The left kidney increased more in length and less in depth than the right so that the areas remained similar as the scale of the variable increased. Kidney depth and area in boys were significantly larger than in girls, both dimensions increasing at equal rates.

Centile charts for each dependent variable by each independent variable were constructed by a non-parametric method.

To discover whether early postnatal ultrasound scanning of the urinary tract might show any evidence of maldevelopment or malfunctionin particular, ureteric reflux-a survey was undertaken of newborn infants delivered at the Princess Mary Maternity Hospital, Newcastle upon Tyne, between 1 January and 31 December 1986. As many of the babies as possible had renal ultrasound scans by a senior radiographer (EWH) between 48 and 72 hours after delivery. Permission for the examination was always sought from the mother, who was given an explanation-both verbally and in writing-of the purpose of the study, the technique to be used, and the steps that would be taken if anything abnormal was found. Approval was obtained from the Newcastle Health Authority ethics committee.

During the project we took the opportunity to measure as many kidneys as possible to try and compile a series of values that could be used in assessing normal (and ultimately, by comparison, abnormal) renal sizes in newborn infants. In this paper we describe the results of the measurements.

Patients and methods

Of a total of 2764 live births during 1986, 1100 mothers $(39 \cdot 8 \%)$ were asked for permission to examine their babies. Twenty three refused, and in a further 15 the examination could not be conducted for technical reasons. A total of 1062 babies were examined. Because of a malfunction in the standoff accessory during the survey, renal measurements were obtained in only 565 .

\section{SCANNING TECHNIQUE}

Babies were usually examined within one hour of a feed so that they would be soothed and relaxed; in many instances they were actually asleep. Apart from the sedative effect, the recent feed ensured a high urine output.

With the baby supine the bladder was scanned to assess its volume. The baby was then rotated into the prone position and after it had settled the kidneys were examined from the posterior aspect. This orientation was used uniformly for both kidneys in all babies even though it was occasionally necessary to make a baby more comfortable by placing it in an oblique or lateral position. The right kidney was usually examined first.

A Hewlett Packard 77020A sector scanner with $5 \mathrm{MHz}$ short and medium focus transducers and a $3.5 \mathrm{~cm}$ standoff accessory was used.

The maximal length (superior to inferior perimeter), depth (superficial to deep perimeter), and area (best fit to circumferential perimeter in longitudinal section) of each kidney were measured twice using two separate frozen images. We also recorded the sex, head circumference, birth weight, and gestational age of the babies and whether they were singletons or multiple births.

\section{Results}

Five babies were excluded from the analysis (two had solitary kidneys, one had a horseshoe kidney, and in two only one kidney was measurable) leaving 560 babies for analysis.

\section{MEASUREMENT ERROR}

In a study of this kind two sources of error will be encountered: natural variation and measurement inconsistency. Because each kidney was measured twice in each dimension it was possible to estimate each source of variation separately. The natural variation is known as the 'between subject' variance and the measurement inconsistency as the 'within subject' variance. As there was little difference between the sexes their results were combined, as were the results for left and right kidneys. Table 1 shows the SD of the measurement inconsistency 
Table 1 Renal size in newborn infants: measurement error and components of variance (total, right and left)

\begin{tabular}{|c|c|c|c|c|}
\hline Dimension & $\begin{array}{l}\text { Between } \\
\text { subject }\end{array}$ & $\begin{array}{l}\text { Within } \\
\text { subject }\end{array}$ & $\begin{array}{l}\text { SD of } \\
\text { measurement } \\
\text { inconsistency }\end{array}$ & $\begin{array}{l}\text { Percentage } \\
\text { total variance } \\
\text { because of } \\
\text { measurement } \\
\text { inconsistency }\end{array}$ \\
\hline $\begin{array}{l}\text { Length }\left(\mathrm{cm}^{2}\right) \\
\text { Depth }\left(\mathrm{cm}^{2}\right) \\
\text { Area }\left(\mathrm{cm}^{4}\right)\end{array}$ & $\begin{array}{l}0 \cdot 194 \\
0.0580 \\
1 \cdot 248\end{array}$ & $\begin{array}{l}0 \cdot 0168 \\
0 \cdot 00607 \\
0 \cdot 101\end{array}$ & $\begin{array}{l}0.13 \mathrm{~cm} \\
0.078 \mathrm{~cm}^{2} \\
0.32 \mathrm{~cm}^{2}\end{array}$ & $\begin{array}{l}7 \cdot 9 \\
9 \cdot 5 \\
7 \cdot 5\end{array}$ \\
\hline
\end{tabular}

and the percentage it contributes to the total variance of the measurements. Considering the difficulties of obtaining organ measurements by ultrasound scanning, particularly in small babies, accuracy was acceptably high.

\section{GENERAL ANALYSIS}

Three independent variables were used (birth weight, gestational age, and head. circumference) to correlate with the three dependent variables (kidney length, kidney depth, and kidney area). A kidney measurement refers to the mean of the two replicates.

Table 2 shows the sex distribution and the means (SD) of the independent variables, and table 3 the means (SD) of the dependent variables.

Kidney size

Table 3 shows that the left kidneys were significantly longer and thinner than the right. Although the differences for length and depth are highly significant, the confidence intervals show that the size of these differences is small (about $1 \mathrm{~mm}$ ).

Association between the means of left and right measurements (mean measurements) and the differences in measurements between sides (left-right) (difference measurements) and sex, birth weight, head circumference, and gestational age

After preliminary analyses, gestational age was abandoned as an independent variable because: the association with kidney size was similar to those of birth weight and head circumference, but generally weaker; it was an extremely skew and coarsely grouped variable so that associa-

Table 2 Renal size in 560 newborn infants: analysis of independent variables

\begin{tabular}{lc}
\hline Sex: & 305 \\
Male & 255 \\
Female & $40(33-42)$ \\
Median (range) gestational age (weeks) & $3291(517)$ \\
Mean (SD) birth weight (g) & $34 \cdot 8(1 \cdot 4)$ \\
Mean (SD) head circumference (cm)* & \\
\hline *Data not available for 12 infants.
\end{tabular}

tions were difficult to detect; and the measurement was likely to be inaccurate, thereby confusing the analysis.

Mean measurements-measurements of depth and area were positively associated with birth weight and head circumference $(p<0.001$ in all cases). Sizes increased at a similar rate in both sexes though male kidneys were slightly larger than female; the difference was stronger with area (birth weight: $p=0.001$, head circumference: $p=0.001$ ) than with depth (birth weight: $p=0.04$, head circumference: $p=0.06$ ) Although significant, the size of the difference between boys and girls was small, roughly $0.6 \mathrm{~mm}$.

Boys differed significantly from girls in the rate at which mean kidney length increased by both birth weight $(p=0.03)$ and head circumference $(p=0 \cdot 01)$, boys being generally larger and increasing faster.

Difference measurements (always left-right)There was no association between length difference measurements and birth weight, head circumference, or sex. For area and depth there was a marginally significant pattern with a tendency for the difference to decrease with increasing birth weight and head circumference in boys, and to increase in girls, though this did not apply to the association between depth and head circumference.

The significance of this finding is unclear. As with the mean measurements, however, the magnitude of the effects is too small to influence the broader assessment of kidney size in newborn infants.

\section{Centile charts}

In newborn infants attention is more likely to be drawn to a kidney that is suspiciously large than to one that is suspiciously small. Conditions such as cystic disease, duplex malformation, hydronephrosis, or neoplasm, are more common in the newborn than hypoplasia or dysplasia. In the construction of the centile charts, therefore, the maximum measurement of the right or left kidney was used. Separate charts for boys and girls were not constructed because this would have roughly halved the number of plots/chart and confused the calculation. Furthermore, the mean difference in measurement between the sexes was small, about the same as the SD of the measurement inconsistencies.

A recently introduced non-parametric method was used. ${ }^{1}$ All the variables were adequately modelled by linear centiles; the intercepts were quadratic and the slopes were linear in the standard normal variate. The models were checked by comparing centile charts for the derived SD scores with those expected from

Table 3 Renal size in 560 newborn infants: analysis of dependent variables

\begin{tabular}{lllll}
\hline Dimension & $\begin{array}{l}\text { Right: } \\
\text { mean }(S D)\end{array}$ & $\begin{array}{l}\text { Left: } \\
\text { mean }(S D)\end{array}$ & $\begin{array}{l}\text { 95\% Confidence interval } \\
\text { of difference between } \\
\text { left and right }\end{array}$ & $\begin{array}{c}p \\
\text { Value }\end{array}$ \\
\hline Length $(\mathrm{cm})$ & $4 \cdot 21(0.45)$ & $4 \cdot 32(0 \cdot 46)$ & 0.08 to $0 \cdot 14$ & $<0 \cdot 001$ \\
Depth $(\mathrm{cm})$ & $2 \cdot 23(0 \cdot 27)$ & $2 \cdot 14(0 \cdot 23)$ & $-0 \cdot 10$ to -0.07 & $<0 \cdot 001$ \\
Area $\left(\mathrm{cm}^{2}\right)$ & $6.48(1 \cdot 19)$ & $6 \cdot 44(1 \cdot 11)$ & -0.11 to 0.03 & 0.29 \\
\hline
\end{tabular}


standard normal variables (MJR Healy, personal communication). The charts refer to the maximum of the means of two replicate measurements made on each kidney.

Centile charts showing $3,10,25,50,75,90$, and 97 centiles were constructed for kidney length and depth by birth weight and head circumference (figs 1-4). Charts for kidney area were also constructed but are not shown.

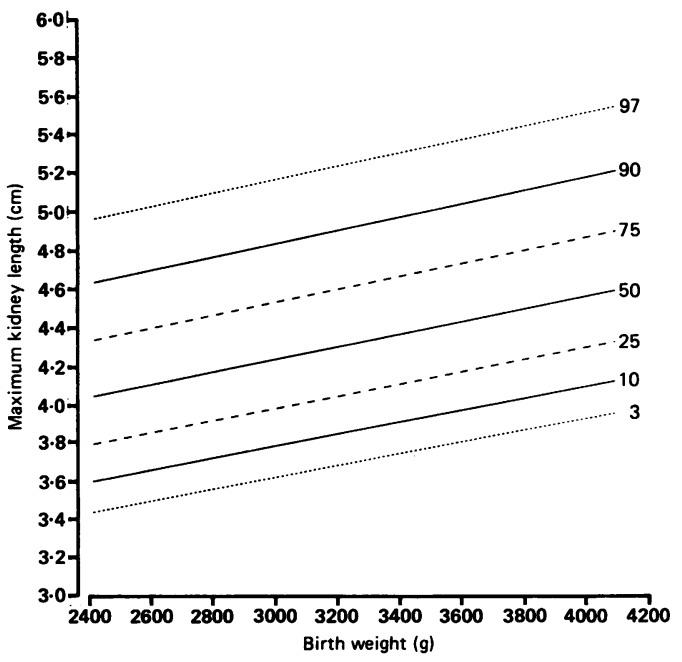

Figure 1 Centile chart: maximum kidney length by birth weight.

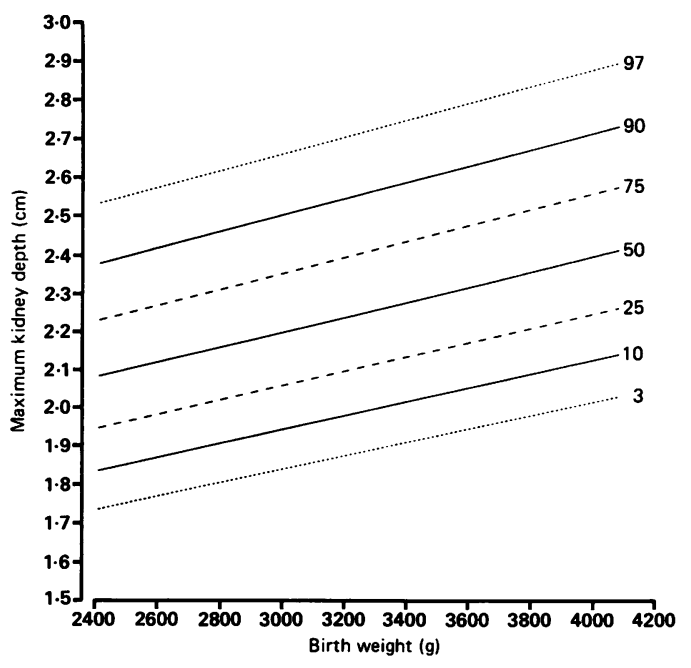

Figure 2 Centile chart: maximum kidney depth by birth weight.

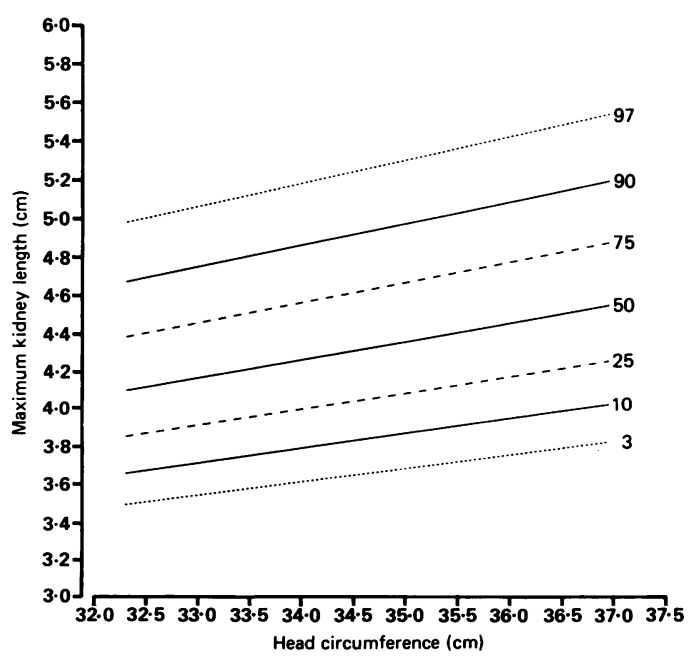

Figure 3 Centile chart: maximum kidney length by head circumference.

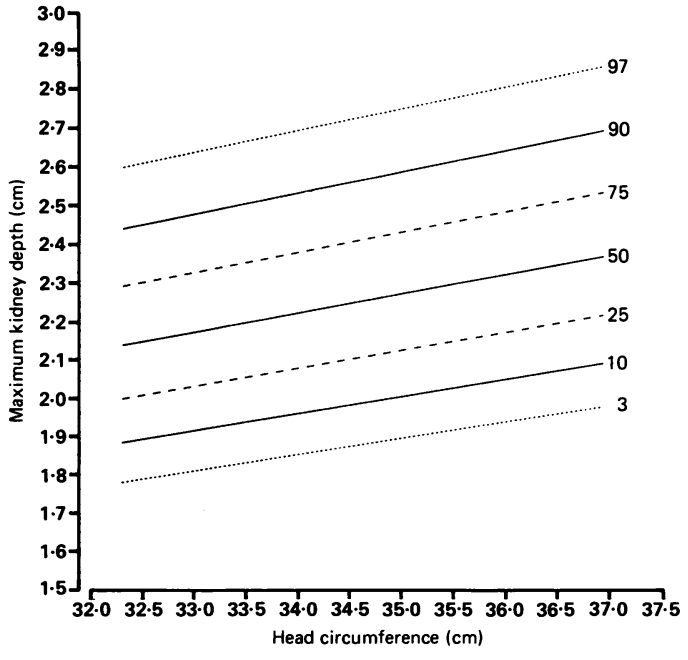

Figure 4 Centile chart: maximum kidney depth by head circumference.

\section{Discussion}

In a study of 100 unselected preterm and term infants renal length was measured by ultrasound scanning, compared with postmenstrual age, and a growth chart plotted. ${ }^{2}$ In 20 of these infants a ratio between kidney length and crownheel measurement was calculated and kidney size remained in proportion to the length of the infant even though the infant was either small or large for gestational age. In study of 36 newborn infants, renal volume and length were measured in order to obtain a mean figure. ${ }^{3}$ There was no correlation with body weight, height, or surface area, but differences in length between right and left kidneys (similar to the current series) were noted, although the volumes were equal. The scanning technique was the same as we used. A comparison between renal length measured by ultrasound and age enabled a regression equation to be calculated, but there were only 10 infants whose mean renal length was roughly equal to the biggest babies described here. ${ }^{4}$ More recently, renal measurements by ultrasound were made in 52 premature infants (all normal size for age) less than 72 hours old; the scanning technique was not described. ${ }^{5}$ No difference in measurement between right and left kidneys was found and correlation between kidney length and birth weight was goodbetter than with body length or body surface area. A regression line with $95 \%$ confidence intervals was plotted. This series was, however, small, and the range of birth weight 530 to $3680 \mathrm{~g}$ was divided into 25 groups. The errors inherent in such a small number might have obscured the difference in length between left and right kidneys.

The measurement of renal dimension in newborn infants is a practical undertaking, and the measurement inconsistency that occurred in this series was low. Because it was necessary to obtain replicate measurements at the same examination, however, the estimate may be slightly too low. Our data suggest that kidney depth is the dependent variable that can most accurately be measured, probably because the ultrasound image of the kidney outline is less curvilinear in this dimension than in others. 
The inconsistency in kidney length measurement was also low. Kidney area measurement is not recommended because the ultrasound scanner calculated this dimension by producing an ellipse that gave the best fit to kidney outline, a technique which is not common to all machines.

The data show a consistent significant difference in shape between the two kidneys; as the babies became larger the left kidney became comparatively longer and thinner, and the right shorter and fatter. It seems likely that the changing shape was caused by the liver, which in the newborn is larger in proportion to the volume of the abdominal cavity than in later life. The right kidney would be compressed longitudinally and expand horizontally thus retaining roughly the same cross sectional area.

There was a significant difference between the sexes in kidney size, boys have larger kidneys than girls in every dimension. The difference was apparent when correlated with both birth weight and head circumference, suggesting that the male fetus has a slightly higher growth velocity than the female and predicting the sex difference in postnatal growth velocity.

The data enable reliable centile charts to be constructed for all three dependent variables by birth weight and head circumference.

Which of the six variables available for correlation are used in the assessment of kidney size in newborn infants will eventually depend on the experience of the operator and the sophistication of the equipment. There is one possible error concerning the use of head circumference: in the rare syndromes associated with atypical head shape (for example, oxycephaly) or more importantly when there has been serious intrauterine growth retardation producing a baby who is 'light for dates' and in whom there is a tendency for 'head sparing', head circumference may be disproportionately large. Kidney centiles in these babies would be smaller when compared with head circumference than birth weight, and it would be neces- sary to take account of both independent variables to obtain a reliable assessment of renal size. In the current series there were only 10 babies who had a gestational age beyond 38 weeks and birth weight below $2500 \mathrm{~g}$, and none of these had a head circumference of more than $35.2 \mathrm{~cm}$, the mean being $33.2 \mathrm{~cm}$. A large discrepancy between expected and actual kidney size in a low birthweight baby would, with other evidence, suggest that there had been intrauterine growth retardation.

\section{CONCLUSION}

It is possible to measure renal size in newborn infants by a standard technique. For increased accuracy a mean of two measurements of length, depth, and area of each kidney should be calculated. A reliable assessment may be obtained by correlating kidney depth or length with birth weight or head circumference, though the latter should be used with caution if intrauterine growth retardation is suspected.

We thank the Newcastle Health Authority Research Committee for financing this project; $M r M$ Flaherty and $\mathrm{Mr}$ S Hall for writing the raw data filing and sorting programme while they were ing the raw data filing and sorting programme while they were Newcastle upon Tyne; Mr G J Flanagan, department of medical Newcastle upon Tyne; Mr G J Flanagan, department of medical physics, University of Newcastle upon Tyne, for transferring the data; the staff members and nurses of the departments of child health and obstetrics, Princess Mary Maternity Hospital, Newcastle upon Tyne, for their cooperation, and finally
Professor M J R Healy for making the software available for fitting the centiles.

1 Healy MJR, Rasbash J, Yang M. Distribution-free estimation of age-related centiles. Ann Hum Biol 1988;15:17-22.

2 De Vries L, Levene MI. Measurement of renal size in preterm and term infants by real-time ultrasound. Arch Dis Child 1983;58:145-7.

3 Holloway $H$, Jones TB, Robinson AE, Harpen MD, Wiseman HJ. Sonographic determination of renal volumes in normal neonates. Pediatr Radiol 1983;13:212-4.

4 Rosenbaum DM, Korngold E, Teele RL. Sonographic assessment of renal length in newborn children. AfR 1984; 142:467-9.

5 Schlesinger AE, Hedlund GL, Pierson WP, Null DM. Normal standards for kidney length in premature infants: Normal standards for kidney length in premature in
determination with US. Radiology 1987;164:127-9. 